Apuntes Universitarios, 2022: 12 (1), enero-marzo

ISSN: 2304-0335 DOI: https://doi.org/10.17162/au.v11i5.917

\title{
Síntomas de ansiedad, conductas agresivas y trastornos alimentarios en adolescentes del norte de Perú
}

\author{
Symptoms of anxiety, aggressive behaviors and eating disorders in \\ adolescents in northern Peru \\ Magaly Quiñones-Negrete ${ }^{1}$, Wendy Arhuis-Inca ${ }^{2}$, Graciela Pérez-Moran ${ }^{3}$, \\ Jovita Coronado-Fernandez ${ }^{4}$, Julio Cjuno ${ }^{51}$ \\ Instituto de Investigación, Capacitación y Desarrollo Psicosocial y Educativo: \\ PSYCOPERU, Perú ${ }^{2}$ \\ Universidad Peruana Unión, Escuela Profesional de Psicología, Lima, Perú ${ }^{35}$ \\ Universidad Cesar Vallejo, Escuela de Medicina, Piura, Perú ${ }^{4}$ \\ iD ORCID ID: https://orcid.org/0000-0001-6772-3041 ${ }^{1}$ \\ iD ORCID ID: https://orcid.org/0000-0001-6772-30412 \\ ORCID ID: https://orcid.org/0000-0002-8497-5686 3 \\ ORCID ID: https://orcid.org/0000-0001-7426-2781 ${ }^{4}$ \\ ORCID ID: https://orcid.org/0000-0001-6732-0381
}

Universidad Católica Los Ángeles de Chimbote, Instituto de Investigación, Chimbote, Perú1

Recibido: 08 de junio de 2021

Aceptado: 02 de setiembre de 2021

\section{Resumen}

Un desorden alimentario podría tener su origen en cualquier factor y estudios previos no determinan con exactitud el riesgo de padecer ansiedad, agresividad como causal de ello. Por tanto, el objetivo de esta investigación fue determinar la relación entre ansiedad y agresividad con los trastornos de conducta alimentaria en adolescentes de la ciudad de Chimbote (Perú). El método utilizado fue el estudio transversal, realizado en una muestra de 916 adolescentes estudiantes de educación secundaria (4to y 5to grado) y universitarios (primer ciclo) en el año 2018. Se calcularon medidas de tendencia central y de dispersión, frecuencias absolutas y relativas. Así también, se realizó la regresión logística binaria para analizar los factores de riesgo para la variable dependiente ajustando las covariables de edad, género, síntomas de ansiedad y conductas agresivas con trastornos de conducta alimentaria, reportado mediante Odd ratios considerando significativo a los $\mathrm{p}<0.05$. Los resultados mostraron que, en promedio, 1 de cada 4 reportaron síntomas de ansiedad, conductas agresivas, $\mathrm{y}$ trastornos de conducta alimentaria por ser de sexo femenino: $\mathrm{OR}=0.66$,

${ }^{1}$ Correspondencia al autor:

E-mail: julio.cjuno@upeu.edu.pe 
tener síntomas de ansiedad $\mathrm{OR}=1.67$ y tener conductas agresivas $\mathrm{OR}=1.72$. representó un riesgo para padecer con trastornos de conducta alimentaria. Se concluye que los síntomas de ansiedad y conductas agresivas representaron un riesgo para padecer con trastornos de conducta alimentaria principalmente en el sexo femenino. Resulta importante que las autoridades del Ministerio de Salud del Perú promuevan un plan de intervención en salud mental en adolescentes para reducir los trastornos de conducta alimentaria como obesidad, sobrepeso y otros.

Palabras clave: Ansiedad, agresividad, anorexia, bulimia, trastorno de conducta alimentaria, alimentación.

\begin{abstract}
An eating disorder could have its origin in any factor, and previous studies do not accurately determine the risk of anxiety, aggressiveness as the cause of it. Therefore, the objective of this research was to determine the relationship between anxiety and aggressiveness with eating disorders in adolescents in the city of Chimbote, Peru. The method used was the cross-sectional study, carried out in a sample of 916 teenage students of secondary education (4th and 5th grade) and university students (first cycle) in 2018. Measures of central tendency and dispersion, absolute and relative frequencies were calculated. Likewise, the binary logistic regression was performed to analyze the risk factors for the dependent variable by adjusting the covariates grouped in age, gender, anxiety symptoms and aggressive behaviors with eating disorders, reported by Odd ratios considering significant at $\mathrm{p}<0.05$. It was found that, on average 1 in 4 reported symptoms of anxiety, aggressive behaviors, and eating disorders due to being female: $\mathrm{OR}=0.66$, have symptoms of anxiety $\mathrm{OR}=1.67$ and have aggressive behavior $\mathrm{OR}=1.72$. It represented a risk for suffering from eating disorders. It is concluded that the symptoms of anxiety and aggressive behaviors represented a risk for suffering from eating disorders mainly in the female gender. It is important that authorities of the Ministry of Health of Peru promote a plan of intervention in mental health in adolescents to reduce eating disorders such as obesity, overweight and others.
\end{abstract}

Keywords: Anxiety, aggressiveness, anorexia, bulimia, eating behavior disorder, nutrition.

\title{
Introducción
}

Los trastornos mentales representan el $16 \%$ de la carga mundial de enfermedades en personas con edades entre 10 y 19 años y los más comunes en esta etapa son la anorexia, bulimia, trastorno por atracón y la adicción a la comida (Organización Mundial de la Salud, 2019). En este caso, se denomina "trastorno alimentario" a cualquier desorden en la conducta alimentaria (comer más o menos de lo normal). Estos trastornos causan alteraciones en el peso y dependencia en algunos casos, lo que origina enfermedades psicológicas, físicas y trastornos sociales que pueden conducir a la muerte. Esta situación afecta 2.5 veces más a mujeres y niñas que a varones y niños, pudiendo aparecer generalmente durante la adolescencia (Figueroa-Quiñones \& Cjuno, 2018; Instituto Nacional de la Salud Mental, 2016). A la fecha se han estudiado estos tipos de trastornos 
en distintos lugares del mundo: Asia, con poblaciones malayas (Tan \& Shashikala, 2019), Colombia, (Fandiño et al., 2007), Paraguay (Ortiz et al., 2017) y Perú (Aliaga-Deza et al., 2010).

Una de las consecuencias de este trastorno viene a ser la ansiedad. Entre los años 2005 al 2015, la ansiedad aumento en un 14,9\%, el mayor caso en mujeres (Organización Mundial de la Salud, 2017). Los países de ingresos bajos son los más propensos a sufrir estos trastornos, siendo reportados en adultos mayores, niños y adolescentes (Organización Mundial de la Salud, 2017). Por otro lado, se define a la ansiedad como la anticipación de un daño o desgracia que se asume sucederá en un futuro, acompañado de sentimientos de disforia y tensión intensa que supera la capacidad adaptativa de la persona; estos síntomas le pueden causar malestar a nivel psicológico, físico y conductual (GuíaSalud, 2008). El National Institute of Mental Health (NIMH) afirma que factores genéticos y ambientales, más la interacción con otros factores como la timidez, ser mujer, tener pocos recursos económicos y la exposición a eventos estresantes, podrían generar mayores probabilidades de padecer algún trastorno de ansiedad (National Institute of Mental Health, 2016).

Otra de las consecuencias del trastorno alimentario es la conducta agresiva que puede manifestarse como un mecanismo de defensa frente a una situación estresante o ansiosa, sea directa o indirectamente y en diversos ámbitos de vida (Ortega \& Mora-Merchán, 1997). Por ejemplo, un estudio en España, en estudiantes de pregrado universitario, revelaron que la ansiedad social relacionada con el estado físico reportó ser positiva y significativamente relacionada $(\mathrm{p}<0.05)$ con la aparición de trastornos alimentarios en adultos jóvenes (Alcaraz-Ibáñez et al., 2019). En el Perú, se han realizado muy pocos estudios relacionados con las variables en estudio, y menos aún existen estudios donde se busque explicar la asociación entre dichas variables. Es por ello, que el presente trabajo tiene como objetivo determinar la relación entre ansiedad y agresividad con los trastornos de conducta alimentaria en estudiantes adolescentes de la ciudad de Chimbote, Perú.

\section{Material y métodos}

\section{Diseño y contexto del estudio}

El presente trabajo consta de un estudio trasversal, utilizando el cuestionario de problemas conductuales en estudiantes de Chimbote en el año 2018. Chimbote es una ciudad-puerto (Figura 1), ubicada en la costa norte del Perú. Cuenta con características étnicas y culturales, y sus habitantes se desempeñan en actividades ligadas a la industria pesquera, la metalurgia, la agricultura y el comercio (Instituto Nacional de Estadística E Informática, 2018). 


\section{Figura 1}

Mapa de ubicación de la ciudad de Chimbote en Perú.

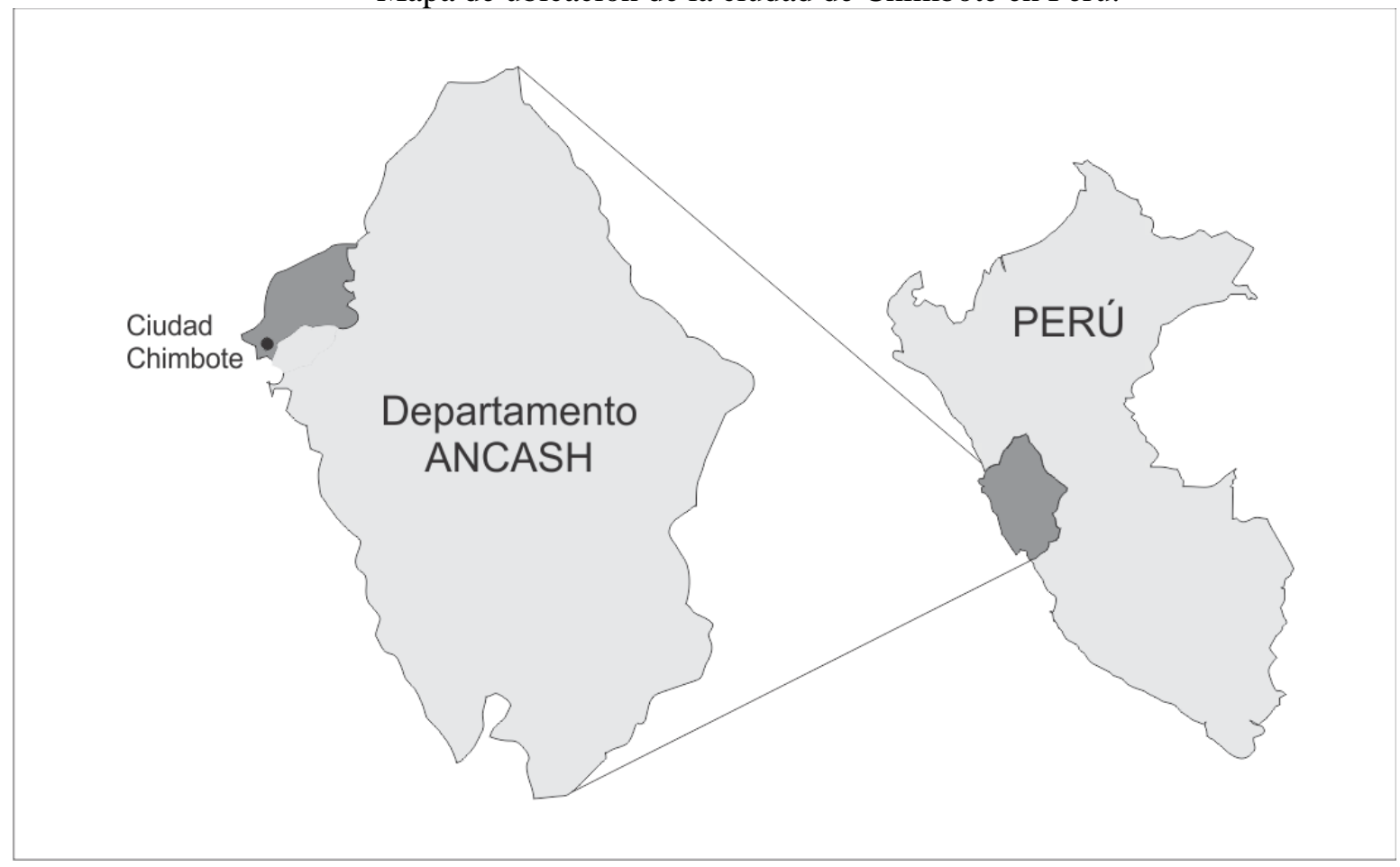

\section{Participantes}

En la evaluación participaron 916 adolescentes con edades entre 15 y 19 años de edad, estudiantes de educación secundaria (4to y 5to grado) y universitarios (primer ciclo) en el año 2018. Se seleccionaron por conveniencia desde un colegio nacional, colegio privado y de una universidad privada de Chimbote. Se excluyeron estudiantes que no podían responder coherentemente la encuesta por presentar algún problema de salud de cualquier índole; así también, aquellos estudiantes cuyos padres no consintieron su participación.

\section{Variables}

La variable dependiente fue "trastornos de conducta alimentaria," la cual se definió como la alteración de la alimentación entre comer menos o más de lo normal. Esta fue evaluada con el cuestionario de prueba de diagnóstico de problemas conductuales o emocionales 2.0. Dichol instrumento pasó por validez de contenido por juicio de seis expertos cuyas especialidades variaban entre psicólogos, médicos y nutricionistas, con experiencia mínima de un año en la evaluación y atención de personas con trastornos de conducta alimentaria. Luego de dos rondas de sugerencias 
de mejora aprobaron la versión final de la encuesta; en seguida, se realizó una prueba piloto con 13 adolescentes con trastornos de conducta alimentaria. Se les aplicó el instrumento y, posteriormente, se organizó un grupo focal para solicitar la sugerencias de mejora sobre la claridad del instrumento. Como siguiente paso, se analizó la confiabilidad por medio de Alfa de Cronbach $(\alpha: 0,71)$ que fue aceptable. Esta variable se categorizó como: sin trastornos y con trastornos.

Las variables independientes fueron: "sexo" (masculino y femenino); "edad" (años cumplidos); "institución educativa" (universidad privada/colegio público/colegio privado), "síntomas de ansiedad" (ausencia/presencia), "conducta agresiva" (ausencia/presencia). Cabe mencionar que la variable "agresividad" fue evaluada por el Cuestionario de Agresividad de Buss y Perry (Chahín-Pinzón et al., 2012). Previamente se evaluó la validez de contenido y cultural por tres jueces expertos con experiencia en psicología educativa. Así también, la variable "síntomas de ansiedad" fue evaluado por la General Anxiety Disorder-7 (GAD-7) (Muñoz-Navarro et al., 2017).

\section{Procedimientos}

La información concluyó en recolectarse en mayo 2018. Asimismo, el personal encargado de la aplicación de las encuestas realizó las siguientes actividades: i) solicitud de los permisos correspondientes a las instituciones educativas, como colegios y universidades que participaron; ii) entrega del asentimiento informado y el consentimiento informado a los padres de todos los estudiantes menores de edad; solo participaron aquellos menores que tenían la autorización firmada y a su vez asintieron en participar en el estudio. Así también, los mayores de edad que firmaron el consentimiento informado; iii) aplicar durante dos semanas la encuesta online a través del formulario de Google, de acceso libre y gratuito. Para el control de los datos se realizó una doble digitación y se cruzó la información hasta tener una versión final de la base de datos en la que aprobaron todos los investigadores, previa verificación aleatoria del 10\% de los datos digitados.

\section{Plan de análisis}

Para el análisis univariado, se determinaron frecuencias relativas y absolutas para las variables categóricas y medidas de tendencia central y de dispersión para las variables numéricas. En el análisis bivariado, se analizó la relación de variable dependiente y la independiente a través de la prueba del chi cuadrado de Pearson, con una potencia del 95\% y un margen de error del 0.05. Mientras, para el análisis multivariado se realizó la regresión logística binaria para analizar los factores de riesgo para la variable dependiente ajustando las covariables "edad agrupada," "sexo," 
"síntomas de ansiedad" y "conductas agresivas con trastornos de conducta," reportado mediante Odd ratios y considerando significativo a los $\mathrm{p}<0.05$. Asimismo, se analizaron los supuestos para el modelo logístico, el mismo cumple con los supuestos de prueba de ómnibus y HosmerLemeshow. El procesamiento y análisis de los datos se realizó en el software estadístico IBM Statistics SPSS 24.0.

\section{Consideraciones éticas}

El estudio fue revisado y aprobado por el Comité Institucional de Ética en Investigación de la Universidad Católica Los Ángeles de Chimbote (Nº13-2018-CEI-VI-ULADECH-Católica). Asimismo, se cumplieron todos los principios éticos de investigación en humanos y en poblaciones vulnerables como son los menores de edad (Ther World Medical Association, 2015).

\section{Resultados}

En el análisis univariado, los adolescentes que participaron en el estudio tenían una edad media de 19 años SD (4.072). En su mayoría 583 (63.6\%) son de sexo femenino; 835 (91.2\%) son estudiantes de una universidad privada; 245 (26.7\%) tienen síntomas de ansiedad; 288 (31.4\%) tienen conductas agresivas; y 224 (24.5\%) tienen trastornos de conducta alimentaria (Tabla 1).

\section{Tabla 1}

Características de estudiantes adolescentes de Chimbote ( $n=916)$

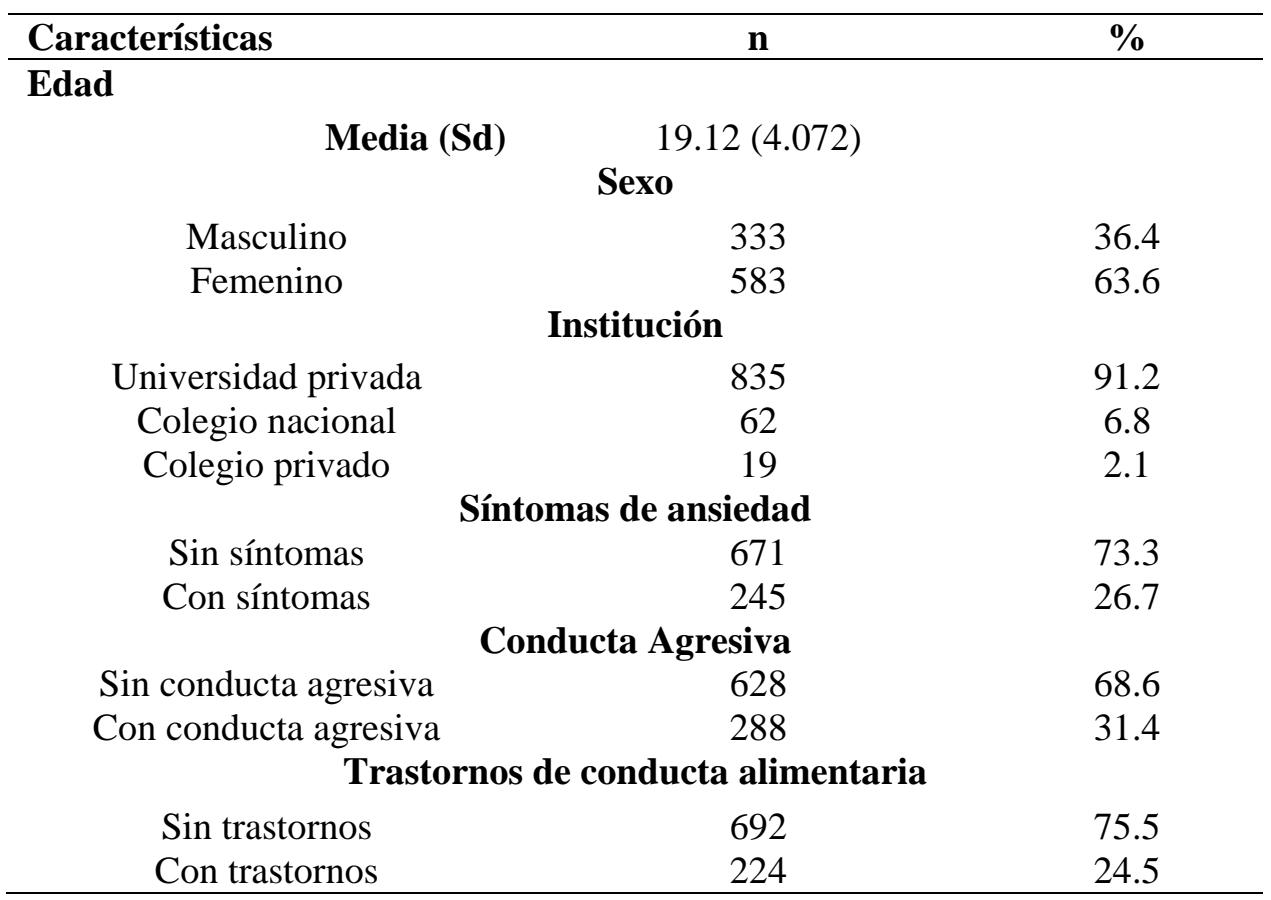


En el análisis bivariado, 159 adolescentes (71.0\%) de sexo femenino y 97 estudiantes (43.3\%) con síntomas de ansiedad mostraron tener mayor prevalencia de trastornos de conducta alimentaria. Además, estas variables se relacionaron significativamente con los trastornos de conducta alimentaria $(\mathrm{p}<0.05)$ (Tabla 2). Así también, 159 participantes $(71.0 \%)$ del sexo femenino y 112 adolescentes (50.0\%) con conducta agresiva mostraron mayor prevalencia de trastorno de conducta alimentaria, y se relacionaron significativamente con trastornos de conducta alimentaria $(\mathrm{p}<0.05)($ Tabla 3$)$.

\section{Tabla 2}

Relación entre edad agrupada, sexo y síntomas de ansiedad con trastornos de conducta alimentaria en estudiantes adolescentes de Chimbote

\begin{tabular}{|c|c|c|c|}
\hline \multirow[t]{2}{*}{ Variables } & \multicolumn{2}{|c|}{ Trastornos de conducta alimentaria } & \multirow[b]{2}{*}{$p$} \\
\hline & $\begin{array}{c}\text { Sin trastornos } \\
(\%)\end{array}$ & $\begin{array}{c}\mathrm{n} \quad \text { Con trastornos } \\
(\%)\end{array}$ & \\
\hline \multicolumn{4}{|l|}{ Sexo } \\
\hline Masculino & $268(38.7)$ & $65(29.0)$ & \multirow{2}{*}{0.002} \\
\hline Femenino & $424(61.3)$ & $159(71.0)$ & \\
\hline \multicolumn{4}{|l|}{ Edad } \\
\hline Mayor de 18 años & $382(55.2)$ & $121(54.0)$ & \multirow{2}{*}{0.408} \\
\hline Menor a 18 años & $310(44.8)$ & $103(46.0)$ & \\
\hline \multicolumn{4}{|l|}{ Institución } \\
\hline Colegio privado & $12(1.7)$ & $7(3.1)$ & \multirow{3}{*}{0.473} \\
\hline Colegio nacional & $47(6.8)$ & $15(6.7)$ & \\
\hline Universidad privada & $633(91.5)$ & $202(90.2)$ & \\
\hline \multicolumn{4}{|l|}{ Síntomas de ansiedad } \\
\hline Con síntomas de ansiedad & $148(21.4)$ & $97(43.3)$ & \multirow{2}{*}{0.000} \\
\hline Sin síntomas de ansiedad & $544(78.6)$ & $127(56.7)$ & \\
\hline
\end{tabular}

$\mathrm{p}=$ valor de significancia del Chi cuadrado. 


\section{Tabla 3}

Relación entre edad agrupada, sexo y conducta agresiva con trastornos de conducta alimentaria en estudiantes adolescentes de Chimbote

\begin{tabular}{lccc}
\hline \multicolumn{1}{c}{ Variables } & \multicolumn{2}{c}{ Trastornos de conducta alimentaria } & \\
\cline { 2 - 3 } & $\begin{array}{c}\text { Sin trastornos } \\
\mathrm{n}(\%)\end{array}$ & $\begin{array}{c}\text { Con trastornos } \\
\mathrm{n}(\%)\end{array}$ & \\
\hline Sexo & & $\boldsymbol{p}$ \\
Masculino & $268(38.7)$ & $65(29.0)$ & \\
Femenino & $424(61.3)$ & $159(71.0)$ & $\mathbf{0 . 0 0 2}$ \\
Edad & $382(55.2)$ & $121(54.0)$ & \\
18 años a más & $310(44.8)$ & $103(46.0)$ & 0.408 \\
Menor a 18 años & $12(1.7)$ & $7(3.1)$ & \\
Institución & $47(6.8)$ & $15(6.7)$ & 0.473 \\
Colegio privado & $633(91.5)$ & $202(90.2)$ & \\
Colegio nacional & $176(25.4)$ & $112(50.0)$ & \\
Universidad privada & $516(74.6)$ & $112(50.0)$ & $\mathbf{0 . 0 0 0}$ \\
Conducta agresiva & & & \\
Con agresividad & & & \\
Sin agresividad & &
\end{tabular}

$\mathrm{p}=$ valor de significancia del Chi cuadrado.

En el análisis multivariado, al ajustar por edad, sexo e institución para determinar la relación entre síntomas de ansiedad y trastornos de conducta alimentaria, tenían más riesgo de padecer trastornos de conducta alimentaria adolescentes de sexo femenino (OR $=0.66$; IC 95\%: .470 $.922 ; \mathrm{p}=0.015)$ y los que tenían síntomas de ansiedad $(\mathrm{OR}=1.67$;C 95\%: $1.416-1.957 ; \mathrm{p}=$ 0.00) (Tabla 4). 


\section{Tabla 4}

Relación por regresión logística binaria entre edad agrupada, sexo, síntomas de ansiedad y trastornos de conducta alimentaria

\begin{tabular}{|c|c|c|}
\hline Variables & OR (IC 95\%) & Sig. \\
\hline \multicolumn{3}{|l|}{ Edad } \\
\hline Menor de 18 años & Ref. & \\
\hline Mayor de 18 años & $1.09(.794-1.523)$ & .566 \\
\hline \multicolumn{3}{|l|}{ Sexo } \\
\hline Masculino & Ref. & \\
\hline Femenino & $.66(.470-.922)$ & .015 \\
\hline \multicolumn{3}{|l|}{ Institución } \\
\hline Universidad privada & Ref. & \\
\hline Colegio nacional & $.50(.185-1.354)$ & .173 \\
\hline Colegio privado & $.59(.187-1.798)$ & .345 \\
\hline \multicolumn{3}{|l|}{ Síntomas de ansiedad } \\
\hline Sin síntomas & Ref. & \\
\hline Con síntomas & $1.67(1.416-1.957)$ & .000 \\
\hline Constante & 0.16 & .000 \\
\hline
\end{tabular}

$\mathrm{OR}=$ Odds ratio, $\mathrm{IC}=$ =Intervalo de confianza, Sig.= significancia, Ref.=categoría de referencia, con negrita las variables con sig. $<0.05$.

Así también, al ajustar por las variables edad, sexo e institución la relación entre conductas agresivas y trastornos de conducta alimentaria, tenían más riesgo de padecer trastornos de conducta alimentaria adolescentes de sexo femenino (OR $=0.62$; IC 95\%: $.442-.872 ; \mathrm{p}=0.006$ ) y los que tenían conductas agresivas $(\mathrm{OR}=1.72 ; \mathrm{IC} 95 \%: 1.468-2.014 ; \mathrm{p}=0.00)($ Tabla 5). 


\section{Tabla 5}

Relación por regresión logística binaria entre edad agrupada, sexo, conductas agresivas y trastornos de conducta alimentaria

\begin{tabular}{|c|c|c|}
\hline Variables & OR (IC 95\%) & Sig. \\
\hline \multicolumn{3}{|l|}{ Edad } \\
\hline Menor de 18 años & Ref. & \\
\hline Mayor de 18 años & $1.10(.796-1.530)$ & .554 \\
\hline \multicolumn{3}{|l|}{ Sexo } \\
\hline Masculino & Ref. & \\
\hline Femenino & $.62(.442-.872)$ & .006 \\
\hline \multicolumn{3}{|l|}{ Institución } \\
\hline Universidad privada & Ref. & \\
\hline Colegio nacional & $.55(.206-1.320)$ & .255 \\
\hline Colegio privado & $.64(.206-2.007)$ & .447 \\
\hline \multicolumn{3}{|l|}{ Conductas agresivas } \\
\hline Sin agresividad & Ref. & \\
\hline Con agresividad & $1.72(1.468-2.014)$ & .000 \\
\hline Constante & .357 & .085 \\
\hline
\end{tabular}

$\mathrm{OR}=$ Odds ratio, $\mathrm{IC}=$ Intervalo de confianza, Sig.= significancia, Ref.=categoría de referencia, con negrita las variables con sig. $<0.05$.

\section{Discusión}

El presente estudio tuvo como objetivo determinar la relación entre ansiedad y agresividad con los trastornos de conducta alimentaria en estudiantes adolescentes de la ciudad de Chimbote. Al respecto, la evaluación de la relación entre edad, sexo, síntomas de ansiedad y trastornos de conducta alimentaria mostró que los adolescentes del sexo femenino $\mathrm{OR}=0.66$, tenían más riesgo de padecer trastornos de conducta alimentaria, seguido de los que tenían síntomas de ansiedad OR $=1.67, \mathrm{y}$ conductas agresivas $\mathrm{OR}=1.72$. Otro hallazgo importante es la prevalencia de los síntomas de ansiedad del 26.7\% (1 de cada 4), el 31.4\% (1 de cada 4) conductas agresivas, y el 24.5\% (1 de cada 4) trastornos de conducta alimentaria. Este resultado coincide con estudios previos donde se reportaron que, ser de sexo femenino y tener síntomas de ansiedad y conductas agresivas reportan mayor prevalencia y riesgo de padecer trastornos de conducta alimentaria (Carolina \& Janet, 2011; Álvarez-Malé, Bautista, \& Serra, 2015; González et al., 2017; Paniagua \& García, 2003). Además, se confirma la asociación entre ansiedad y trastorno de conducta alimentaria (Alvarez-Malé et al., 2015: Behar et al., 2007). Sobre la causa, el informe de la UNICEF señala que los adolescentes son más propensos a sufrir estos tipos de enfermedades por estereotipos del mundo actual (Fondo de las Naciones Unidas para la Infancia, 2011). 
El trastorno alimentario es una enfermedad muy seria, y se encuentra asociada a una significativa morbilidad y mortalidad, que ha ido incrementando en los países en desarrollo (Kolar et al., 2016). Esto confirma que los trastornos de conducta alimentaria más frecuentes en la adolescencia son: la anorexia nerviosa, bulimia nerviosa, trastorno por atracón y otros trastornos alimentarios y de la ingestión de alimentos especificados (Gaete el al., 2020). Por otro lado, Ramírez (2021) destaca el riesgo de presentar ansiedad cuando existe un desorden alimentario, las manifestaciones de fobia en los cuadros alimentarios son el temor a las situaciones sociales que impliquen el comer, y que el propio cuerpo sea sometido a escrutinio por los demás, con miedo a hablar, a sonrojarse, a comer o beber en público, y a hacer el ridículo, por sentimientos abrumadores de baja autoestima, rechazo a su figura y menosprecio de sí mismas. Esto debido a que, en la etapa de la adolescencia, existen cambios físicos y cognitivos que deben enfrentar los adolescentes de acuerdo a la demanda que pueda existir en su contexto sociocultural, y ello dependerá cómo los adolescentes pueden enfrentarla o ser víctima de este tipo de trastornos.

Por otra parte, los riesgos durante la adolescencia se asocian a las condiciones de su desarrollo humano, y que podrían estar generando una preocupación excesiva, evitándoles llevar una vida normal, dificultades para controlar sus preocupaciones, síntomas de inquietud o sensación de estar atrapado, y dificultades para concentrarse (American Psychiatric Association, 2014), que podrían estar conduciendo a generar un trastorno en la conducta alimentaria como un medio de liberación de la tensión emocional. En este caso, estudios previos en Colombia (Cardona-Arias et al., 2015) mostraron que 76.2\% adolescentes reportaron un estado ansioso (Arrieta-Vergara et al., 2014) y en Madrid un 15,2\% (Garcia et al., 2018) presentaron prevalencias de síntomas de ansiedad, que en general son variantes, pero muestran que esta población sí presenta prevalencias considerables de ansiedad. Los trastornos de conducta alimentaria se inician habitualmente en la adolescencia, y se asocian con problemas psicológicos como: el aislamiento social, dificultades para fortalecer el auto concepto, la autoestima, la autonomía y la capacidad de intimar, por lo que estas enfermedades pueden interferir en el logro de las tareas propias de la adolescencia (Hornberger \& Lane, 2021). También, refiere que es muy común la comorbilidad psiquiátrica, especialmente los síntomas depresivos, los trastornos ansiosos, el trastorno obsesivo-compulsivo, y los comportamientos auto agresivos (Nagata et al., 2020; Ridout et al., 2021).

Por otro lado, la prevalencia de agresividad que se encontró en 31.4\% (1 de cada 4) es más alta, en comparación con los adolescentes españoles $13.8 \%$, mexicanos $16.4 \%$, chinos $15.7 \%$ 
(Torregrosa et al., 2010) y 18\% colombianos (Castillo, 2013). Aquello podría estar asociado a un estado ansioso y a su condición de adolescente que desafía por naturaleza cualquier situación que le parece injusta. Esto conduce a mostrar conductas agresivas como una forma de liberar el estado ansioso, que puede ser desencadenada por la necesidad de consumir o evitar el consumo de alimentos (Chung et al., 2019). Al respecto, el Ministerio de Salud de Perú ( 2017) afirma que los problemas alimentarios tienen mayor prevalencia en las mujeres (7.1\%) que los varones (4.9\%) Otro estudio, realizado en Cantabria, España, mostró que el 9.1\% adolescentes de sexo femenino presentan signos de alerta de trastornos de conductas alimentarias (Paniagua Repetto \& García Calatayud, 2003).

Finalmente, una limitación del estudio es que los datos fueron obtenidos en poblaciones de adolescentes escolares y universitarios; las diferencias de formación educativa podrían haber influido en las altas prevalencias de las variables de estudio, pero por estar dentro de una etapa de desarrollo integral como es la adolescencia, el presente resultado no deja de tener valor. Otra limitación sería que el uso de instrumentos validados por el mismo equipo a pesar de la existencia de instrumentos psicométricos ya validados para ansiedad y agresividad. No obstante, los propios instrumento de ansiedad (.74), de agresividad (.67) y de trastornos de conducta alimentaria (.71) mostraron grados de confiabilidad aceptables; por lo que los resultados de este estudio son importantes y válidos.

\section{Conclusión}

Ser de sexo femenino, tener síntomas de ansiedad y tener conductas agresivas, representó un riesgo para padecer con trastornos de conducta alimentaria en adolescentes de Chimbote. 1 de cada 4 adolescentes presentaron trastornos de conducta alimentaria. Por ello, resulta importante que la autoridad del Ministerio de Salud del Perú promueva un plan de intervención en salud mental en adolescentes para reducir los trastornos de conducta alimentaria que contribuye a la población de los adolescentes con sobrepeso y obesidad del Perú, así como a la población con bulimia y anorexia.

\section{Referencias}

Alcaraz-Ibáñez, M., Sicilia, Á., \& Paterna, A. (2019). Exploring the differentiated relationship between appearance and fitness-related social anxiety and the risk of eating disorders and 
depression in young adults. Scandinavian Journal of Psychology, 60 (6), 569-576. https://doi.org/10.1111/sjop.12584

Aliaga-Deza, L., La Cruz-Saldaña, T., \& Vega-Dienstmaier, J. (2010). Sintomatología de los trastornos de la conducta alimentaria en adolescentes de un colegio del distrito de Independencia, Lima, Perú. Revista de Neuro-Psiquiatría, 73 (2), 52-61. http://www.redalyc.org/articulo.oa?id=372036930004

Alvarez-Malé, M. L., Bautista Castaño, I., \& Serra Majem, L. (2015). Prevalencia de los trastornos de la conducta alimentaria en adolescentes de Gran Canaria. Nutrición Hospitalaria, 31 (5), 2283-2288. https://doi.org/10.3305/nh.2015.31.5.8583

American Psychiatric Association. (2014). Guía de consulta de los criterios diagnósticos del DSM5 (5th ed.). https://areaclinicapediatrica.files.wordpress.com/2016/03/d5-completo-enespac3b1ol.pdf

Arrieta Vergara, K. M., Díaz Cárdenas, S., \& González Martínez, F. (2014). Síntomas de depresión y ansiedad en jóvenes universitarios: prevalencia y factores relacionados. Revista Clínica de Medicina de Familia, 7 (1), 14-22. https://doi.org/10.4321/s1699-695x2014000100003

Behar A, R., Barahona I, M., Iglesias M, B., \& Casanova Z, D. (2007). Trastorno depresivo, ansiedad social y su prevalencia en trastornos de la conducta alimentaria. Revista chilena de neuro-psiquiatría, 45 (3), 211-220. https://doi.org/10.4067/S0717-92272007000300005

Cardona-Arias, J. A., Pérez-Restrepo, D., Rivera-Ocampo, S., Gómez-Martínez, J., \& Reyes, Á. (2015). Prevalence of anxiety in universitary students. Perspect. Psicol, 11 (1), 079-089. http://www.scielo.org.co/pdf/dpp/v11n1/v11n1a06.pdf?fbclid=IwAR20sJzL2MiNPcvr8HcZ2OMNuzqkqqAKbslbVEe96K-bgetMQLBwaq-roE

Carolina, L. G., \& Janet, T. (2011). Trastornos de la conducta alimentaria en adolescentes: descripción y manejo. Revista Médica Clínica Las Condes, 22 (1), 85-97. https://doi.org/10.1016/S0716-8640(11)70396-0

Castillo, M. (2013). Manifestaciones de la Conducta de Agresión en el Contexto Universitario. Escenarios, 11 (1), 139-148. http://ojs.uac.edu.co/index.php/escenarios/article/view/188 Chahín-Pinzón, N., Lorenzo-Seva, U., \& Vigil-Colet, A. (2012). Características psicométricas de la adaptación colombiana del Cuestionario de Agresividad de Buss y Perry en una muestra de preadolescentes y adolescentes de Bucaramanga. Universitas Psychologica, 11 (3), 979-988. http://www.scielo.org.co/scielo.php?script=sci_arttext\&pid=S1657-92672012000300025 
Chung, J., Song, G., Kim, K., Yee, J., Kim, J., Lee, K., \& Gwak, H. (2019). Association between anxiety and aggression in adolescents: a cross-sectional study. BMC pediatrics, 19 (1). https://doi.org/10.1186/S12887-019-1479-6

Fandiño, A., Giraldo, S., Martinez, C., Aux, C. P., \& Espinosa, R. (2007). Factores asociados con los trastornos de la conducta alimentaria en estudiantes universitarios en Cali, Colombia. Colombia Médica, 38 (4), 27. https://www.redalyc.org/pdf/283/28338402.pdf

Figueroa Quiñones, J., \& Cjuno, J. (2018). Adicción alimentaria en Latinoamérica. Medwave, 1818 (11). https://doi.org/10.5867/medwave.2018.01.7171

Fondo de las Naciones Unidas para la Infancia. (2011). Estado mundial de la infancia 2011. La adolescencia: Una época de oportunidades. https://www.cepal.org/es/notas/estado-mundialla-infancia-2011-la-adolescencia-epoca-oportunidades

Garcia, J. M., Lacalle, M., Valbuena, M. C., \& Polaino, A. (2018). Educación y relaciones familiares: ansiedad, depresión y otras variables. Revista Internacional Jurídica y Empresarial., 1. https://portalderevistas.ufv.es/rije/article/view/292/288

González Quiñones, J. C., Martínez Caro, D. C., Martínez Muñoz, S. G., \& Pinzón Ramírez, J. A. (2017). Relación entre trastorno de conducta alimentaria y género y familia en adolescentes escolarizados, Suba (Bogotá). Carta Comunitaria, $25 \quad$ (143), 29. https://doi.org/10.26752/ccomunitaria.v25.n143.81

GuíaSalud. (2008). Guía de Práctica Clínica sobre Transtornos de Ansiedad en Atención Primaria. Versión completa. Definición, manifestaciones clínicas y clasificaciones. GuíaSalud. es. http://www.guiasalud.es/egpc/ansiedad/completa/apartado04/definicion_diagnostico.html

Hornberger, L., \& Lane, M. (2021). Identification and Management of Eating Disorders in Children and Adolescents. Pediatrics, 147 (1). https://doi.org/10.1542/PEDS.2020-040279

Instituto Nacional de Estadística E Informática. (2018). Perú: Perfil Sociodemográfico - Informe $\begin{array}{llll}\text { Nacional } & - & \text { Censos } & \text { Nacionales }\end{array}$ https://www.inei.gob.pe/media/MenuRecursivo/publicaciones_digitales/Est/Lib1539/libro.p df

Instituto Nacional de la Salud Mental. (2016). Los trastornos de la alimentación: Un problema que va más allá de la comida. https://www.nimh.nih.gov/health/publications/espanol/lostrastornos-de-la-alimentacion/str-16-4901_152220.pdf

Ministerio de Salud. (2017). Documento técnico Situación de Salud de los Adolescentes y jóvenes 
en el Perú 2017. Cincoeditores. http://bvs.minsa.gob.pe/local/MINSA/4143.pdf

Muñoz-Navarro, R., Cano-Vindel, A., Moriana, J., Medrano, L., Ruiz-Rodríguez, P., AgüeroGento, L., Rodríguez-Enríquez, P., Pizà, M., \& Ramírez-Manent, J. (2017). Screening for generalized anxiety disorder in Spanish primary care centers with the GAD-7. Psychiatry research, 256, 312-317. https://doi.org/10.1016/J.PSYCHRES.2017.06.023

Nagata, J., Ganson, K., \& Murray, S. (2020). Eating disorders in adolescent boys and young men: an update. Current opinion in pediatrics, 32 (4), 476-481. https://doi.org/10.1097/MOP.0000000000000911

National Institute of Mental Health. (2016). Anxiety Disorders. NIH. https://www.nimh.nih.gov/health/topics/anxiety-disorders/index.shtml

Organización Mundial de la Salud. (2017). Depresión y otros trastornos mentales comunes Estimaciones sanitarias mundiales. http://iris.paho.org/xmlui/bitstream/handle/123456789/34006/PAHONMH17005-spa.pdf

Organización Mundial de la Salud. (2019). Salud mental del adolescente. WHO. https://www.who.int/es/news-room/fact-sheets/detail/adolescent-mental-health

Ortega, R., \& Mora-Merchán, J. A. (1997). Agresividad y violencia. El problema de la victimización entre escolares. Revista de Educación, 313, 7-27.

Ortiz, L., Aguiar, C., Samudio, G., \& Troche, A. (2017). Trastornos de la conducta alimentaria en adolescentes: una patología en auge? Pediatr. (Asunción), 44 (1), 37-42. https://www.revistaspp.org/index.php/pediatria/article/view/151/146

Paniagua Repetto, H., \& García Calatayud, S. (2003). Signos de alerta de trastornos alimentarios, depresivos, del aprendizaje y conductas violentas entre adolescentes de Cantabria. Revista Española de Salud Pública, 77 (3), 411-422. https://doi.org/10.1590/S113557272003000300011

Ridout, S., Ridout, K., Kole, J., Fitzgerald, K., Donaldson, A., \& Alverson, B. (2021). Comparison of eating disorder characteristics and depression comorbidity in adolescent males and females: $\begin{array}{llrl}\text { An observational } & \text { study. } & \text { Psychiatry } & \text { research, }\end{array}$ https://doi.org/10.1016/J.PSYCHRES.2020.113650

Tan, J. J., \& Shashikala, S. (2019). Body Dissatisfaction and Risk of Eating Disorder among UCSI University Non-Science Field Students. Indian Journal of Public Health Research \& Development, 
http://www.indianjournals.com/ijor.aspx?target=ijor:ijphrd\&volume=10\&issue $=4 \&$ article $=2$ 09

Ther World Medical Association. (2015). Declaración de Helsinki de la AMM - Principios éticos para las investigaciones médicas en seres humanos. WMA. https://www.wma.net/es/policiespost/declaracion-de-helsinki-de-la-amm-principios-eticos-para-las-investigaciones-medicasen-seres-humanos/

Torregrosa, M. S., Inglés, C. J., García-Fernández, J. M., Ruiz-Esteban, C., López-García, K. S., \& Zhou, X. (2010). Diferencias en conducta agresiva entre adolescentes españoles, chinos y mexicanos. European Journal of Education and Psychology, 3 (2), 167-176. https://www.redalyc.org/pdf/1293/129315468001.pdf

United Nations Educational Scientific and Cultural Organization. (2019). Behind the numbers: ending school violence and bullying. http://www.infocoponline.es/pdf/ENDING-SCHOOLVIOLENCE.pdf 